\title{
INTERVENÇÕES FARMACÊUTICAS DESTINADAS À OTIMIZAÇÃO DA ADESÃO AO TRATAMENTO MEDICAMENTOSO DE UM PACIENTE
}

\author{
INTERVENTION FOR THE PHARMACEUTICAL DRUG TREATMENT \\ OPTIMIZATION TO MEMBERSHIP OF A PATIENT
}

INTERVENCIÓN PARA LA OPTIMIZACIÓN DE TRATAMIENTO DE DROGAS FARMACÉUTICAS PARA MIEMBROS DE UN PACIENTE

\section{Rinaldo Eduardo Machado Oliveira ${ }^{1 *}$, Marina Del Vecchio Filipin ${ }^{2}$, Mariana Honorato Giardini ${ }^{2}$}

\footnotetext{
${ }^{1}$ Faculdade de Medicina de Ribeirão Preto - Universidade de São Paulo

${ }^{2}$ Hospital Estadual de Ribeirão Preto

E-mail*: rinaldo_eduardo@hotmail.com
}

Submetido em: 08/03/2015; Aceito em: 13/05/2015; Publicado em: 30/06/2015

\section{RESUMO}

Objetivo: Descrever as intervenções farmacêuticas realizadas em conjunto à equipe multiprofissional para otimizar a adesão ao tratamento medicamentoso de um paciente idoso. Métodos: Estudo de caso realizado em um Hospital Público de Atenção Secundária com coleta de dados a partir de prontuário eletrônico, teste de adesão BMQ e consulta ao Micromedex ${ }^{\circledR}$. Resultados: Paciente analfabeto, com potencial baixa adesão ao tratamento medicamentoso, devido a problemas no uso correto e acesso na rede pública de saúde. Os farmacêuticos analisaram aspectos da terapia farmacológica e identificou as principais causas da baixa adesão ao tratamento. Posteriormente, foram apresentadas 
OLIVEIRA, R.E.M.; FILIPIN, M.D.V; GIARDIN, M.H.

REF-ISSN1808-0804 Vol.XII (2), 39-51, 2015.

estratégias para otimizar a utilização de medicamentos juntamente com a equipe multiprofissional e garantia da continuidade do cuidado pós-internação na Atenção Primária à Saúde através do Núcleo de Apoio à Saúde da Família do município de origem do paciente. Conclusão: As ações farmacêuticas destinadas à melhoria da adesão ao tratamento medicamentoso do paciente idoso tornam-se essencial na atenção hospitalar, garantindo efetividade da assistência prestada. O hospital é um importante espaço de promoção e educação em saúde.

Palavras-chave: Assistência Hospitalar, Idoso, Equipe de Assistência ao Paciente

\section{ABSTRACT}

Objective: Describe the pharmaceutical interventions together the multidisciplinary team to optimize adherence to drug treatment of an elderly patient. Methods: Case report conducted in a Public Hospital Secondary Care with collecting data from electronic medical records, membership BMQ test and consultation to Micromedex $®$. Results: Patient illiterate, with potential poor adherence to drug treatment, due to problems in the correct use and access in public health. Pharmacists analyzed aspects of drug therapy and identified the main causes of poor adherence to treatment. Subsequently, strategies were presented to optimize the use of medications along with the multidisciplinary team and ensuring the continuity of post-hospital care in primary health care through the Support Center for Family Health in the municipality of origin of the patient. Conclusion: Drug actions aimed at improving the adherence to medication of elderly patients become essential in hospital care, ensuring effectiveness of care provided. The hospital is an important area of promotion and health education.

Keywords: Hospital Care, Aged, Patient Care Team

\section{RESUMEN}

Objetivo: Describir las intervenciones farmacéuticas junto al equipo multidisciplinario para optimizar la adherencia al tratamiento farmacológico de un paciente de edad avanzada. Métodos: Reporte de un caso realizado en un Hospital Público de Atención secundaria con la recolección de datos de la historia clínica electrónica, la membresía de prueba BMQ y consulta a Micromedex $®$. Resultados: Paciente analfabeta, con potencial de falta de adherencia al tratamiento farmacológico, debido a problemas en el correcto 
OLIVEIRA, R.E.M.; FILIPIN, M.D.V; GIARDIN, M.H.

REF-ISSN1808-0804 Vol.XII (2), 39-51, 2015.

uso y el acceso a la salud pública. Los farmacéuticos analizaron aspectos de la terapia con medicamentos e identificaron las principales causas de la falta de adherencia al tratamiento. Posteriormente, se presentaron las estrategias para optimizar el uso de medicamentos junto con el equipo multidisciplinario y la garantía de la continuidad de la atención post-hospitalaria en la atención primaria de la salud a través del Centro de Apoyo a la Salud de la Familia en el municipio de origen del paciente. Conclusione: Las acciones de la droga dirigidos a mejorar la adherencia a la medicación de los pacientes de edad avanzada se vuelven esenciales en la atención hospitalaria, lo que garantiza la eficacia de la atención prestada. El hospital es una importante área de promoción y educación para la salud.

Palabras clave: Atención Hospitalaria, Anciano,Grupo de Atención al Paciente

\section{INTRODUÇÃO}

O aumento do número de idosos é um fenômeno mundial que afeta tanto países desenvolvidos quanto em desenvolvimento ${ }^{(1)}$. No Brasil, o processo de mudanças demográficas é acompanhado por transformações epidemiológicas com alterações relevantes no quadro de morbimortalidade. Observa-se redução das taxas de doenças infecciosas e parasitárias e predomínio de doenças crônicas não transmissíveis ${ }^{(2)}$. Tais doenças exigem acompanhamento por equipes multiprofissionais permanentes, intervenções contínuas e uso de medicamentos. Logo, é importante haver adesão do paciente ao regime terapêutico prescrito $^{(3)}$.
A boa adesão depende de um conjunto de fatores, dentre os quais se destacam: relação profissional da saúdepaciente, crenças individuais do paciente, aspectos relacionados ao tratamento, à doença, ao acesso aos serviços de saúde e aos medicamentos, bem como o suporte familiar. A não adesão ao tratamento medicamentoso pode gerar diversas complicações, levando a hospitalização com aumento dos custos ao sistema de saúde ${ }^{(4)}$.

A equipe multiprofissional, em âmbito hospitalar, deve utilizar estratégias educacionais para desenvolvimento do autocuidado durante a internação. Associado a isso, um planejamento do cuidado antes da alta, poderá ter impacto importante no comportamento dos pacientes com 
OLIVEIRA, R.E.M.; FILIPIN, M.D.V; GIARDIN, M.H.

REF-ISSN1808-0804 Vol.XII (2), 39-51, 2015.

consequente redução das taxas de não adesão ao tratamento ${ }^{(5)}$.

O presente estudo de caso objetiva descrever as intervenções farmacêuticas realizadas em conjunto à equipe multiprofissional em um Hospital de Atenção Secundária para otimizar a adesão ao tratamento medicamentoso de um paciente internado com complicações devido má adesão ao tratamento proposto para controle da Hipertensão Arterial Sistêmica (HAS) e outras comorbidades.

\section{MÉTODOS}

A coleta de dados foi realizada através do prontuário eletrônico da instituição, prescrições médicas, exames clínico-laboratoriais, intervenções da equipe multiprofissional e monitorização do paciente.

O questionário utilizado para avaliação da adesão ao tratamento medicamentoso na perspectiva do paciente foi $\mathrm{O}$ Brief Medication Questionaire (BMQ). Este instrumento identifica barreiras à adesão em três domínios: 1) regime; 2) crenças e 3 ) recordação(6). Utilizou-se a versão do BMQ traduzida para a língua portuguesa e validado que classifica a adesão de acordo com o número de resposta positivas em: alta adesão (nenhuma), provável adesão (1), provável baixa adesão (2) e baixa adesão (3 ou mais) em qualquer domínio. Foi considerado o uso de medicamentos nos sete dias precedentes à internação.

A identificação de possíveis interações medicamentosas foi realizada através da consulta a base de dados Micromedex ${ }^{\circledR(7)}$. Através do ofício $n^{\circ}$ 3620/2014 o Comitê de Ética em Pesquisa do Hospital das Clínicas da Faculdade de Medicina de Ribeirão Preto da Universidade de São Paulo tornou-se ciente do presente relato.

\section{RELATO DE CASO}

Paciente sexo feminino, 66 anos, viúva e analfabeta. Possui quatro filhos, sendo um deficiente físico, um deficiente mental e os outros dois casados. Proveniente de uma cidade do interior de Minas Gerais e realiza acompanhamento ambulatorial em um Hospital Público de Atenção Terciária. Durante consulta médica de rotina apresentou-se assintomática, pressão arterial $(P A)=$ $200 \times 100 \mathrm{mmHg}$ e relato de uso irregular dos medicamentos em domicílio. Foi encaminhada a um Hospital Público de Atenção Secundária para controle pressórico. 
OLIVEIRA, R.E.M.; FILIPIN, M.D.V; GIARDIN, M.H.

REF-ISSN1808-0804 Vol.XII (2), 39-51, 2015.

As hipóteses diagnósticas referidas pelo médico no momento da internação eram: HAS, hipotireoidismo, Diabetes mellitus (DM) tipo 2, obesidade, dislipidemia, insuficiência cardíaca congestiva (ICC) e insuficiência renal crônica (IRC).
Durante a internação foram realizados o monitoramento da pressão arterial sistólica (PAS) e pressão arterial diastólica (PAD) (Figura 1) e exames laboratoriais nos dias D2, D4, D7 (Tabela 1).

Figura 1 - Representação gráfica das variações dos valores de pressão arterial sistólica e pressão arterial diastólica $(\mathrm{mmHg})$ em função dos dias de internação (D1 a D9). Estão representados os pontos com mediana, valores mínimo e máximo.

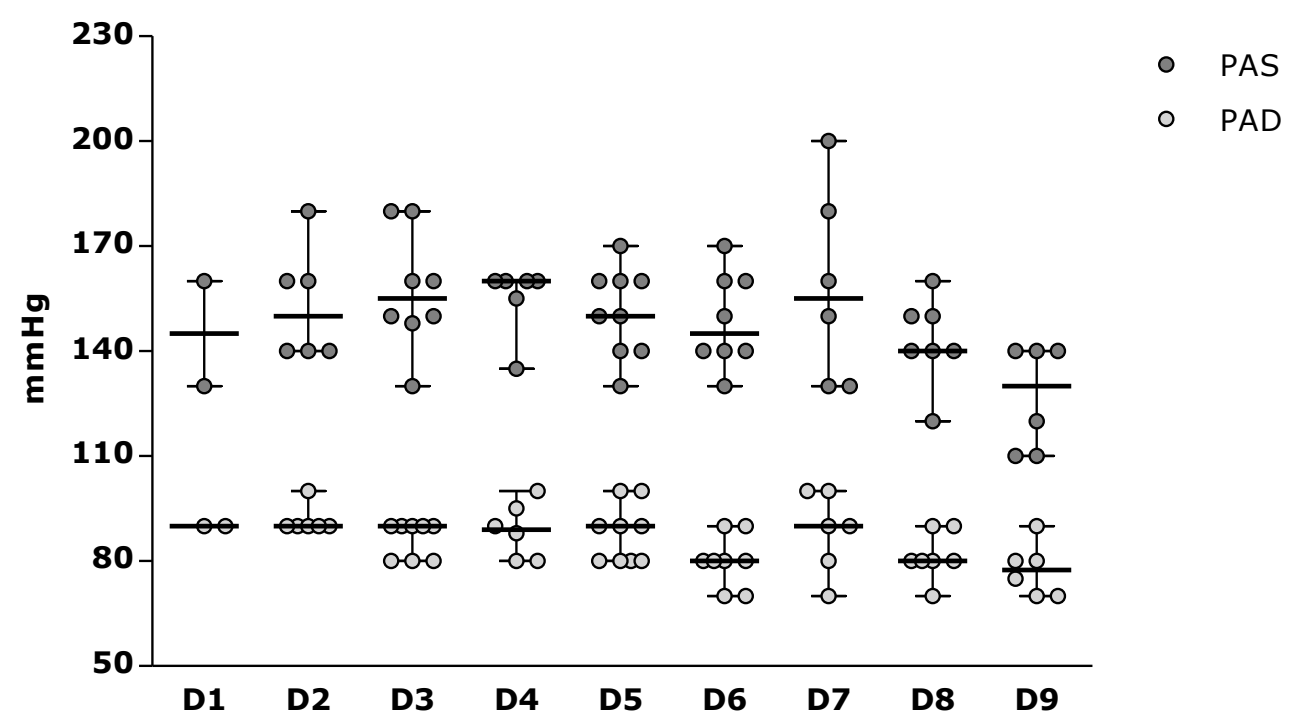

Dias de internação 
OLIVEIRA, R.E.M.; FILIPIN, M.D.V; GIARDIN, M.H.

REF-ISSN1808-0804 Vol.XII (2), 39-51, 2015.

Tabela 1 - Resultados dos exames laboratoriais solicitados durante os dias da internação.

\begin{tabular}{c|c|c|c}
\hline $\begin{array}{c}\text { Dia de } \\
\text { internação }\end{array}$ & D2 & D4 & D7 \\
\hline Sxádio $(\mathbf{m m o l} / \mathbf{L})$ & 136 & 135 & 137 \\
\hline Potássio $(\mathbf{m m o l} / \mathbf{L})$ & 4 & 4,30 & 4,90 \\
\hline Creatinina $(\mathbf{m g} / \mathbf{d L})$ & 1,11 & 1,20 & 1,35 \\
\hline Ureia $(\mathbf{m g} / \mathbf{d L})$ & 55 & 51 & 60 \\
\hline TSH $(\boldsymbol{\mu} \mathbf{U} / \mathbf{m L})$ & 4,10 & - & - \\
\hline
\end{tabular}

Conforme rotina do Serviço de Assistência Farmacêutica do Hospital foi executada a conciliação dos medicamentos, verificação de dose, posologia e análise das interações medicamentosas. No atendimento farmacêutico ao paciente foram fornecidas orientações gerais do tratamento, avaliação da adesão, acesso aos medicamentos e esclarecimentos de dúvidas.

A potencial má adesão ao tratamento foi detectada através do BMQ cujo escore foi 6-0-2 e não foi identificada nenhuma interação medicamentosa classificada como grave.

Dentre as condutas do seguimento farmacêutico, realizou-se reunião entre os filhos do paciente (aqueles sem limitações físicas e mentais) e equipe multiprofissional formada por médicos, farmacêuticos e assistente social. Nesta reunião a equipe apresentou o quadro clínico do paciente, dificuldades na adesão ao tratamento e a importância da responsabilização da família no cuidado. A família apresentouse receptiva comprometendo-se a colaborar com as necessidades do paciente.

Visando a integralidade da assistência, os farmacêuticos do Hospital articularam a continuidade do seguimento farmacêutico com a equipe do Núcleo de Apoio à Saúde da Família 
OLIVEIRA, R.E.M.; FILIPIN, M.D.V; GIARDIN, M.H.

REF-ISSN1808-0804 Vol.XII (2), 39-51, 2015.

(NASF) do município de origem do do município de origem do paciente, com paciente.

Após estabilização do quadro clínico a equipe médica revisou os medicamentos para utilização em domicílio após a internação (Tabela 2). Os medicamentos prescritos na alta são idênticos aos utilizados antes da internação, entretanto, houve ajustes nas doses. Os medicamentos são adquiridos na Farmácia da Rede Básica exceção da Atorvastatina que é adquirida através do Componente Especializado na Assistência Farmacêutica conforme critérios de inclusão ao protocolo e Bromazepam que é adquirido com recursos financeiros do paciente. Com o objetivo de organizar os medicamentos prescritos, os farmacêuticos forneceram dispositivos identificando os horários de administração.

Tabela 2 - Medicamentos prescritos na alta-hospitalar.

\begin{tabular}{|c|c|}
\hline Medicamentos & Posologia \\
\hline Carvedilol 25 mg & $50 \mathrm{mg} / \mathrm{dia}$ via oral \\
\hline Isossorbida $10 \mathrm{mg}$ & 60 mg/dia via oral \\
\hline Furosemida 40 mg & 40 mg/dia via oral \\
\hline Clonidina 150 mcg & $600 \mathrm{mcg} /$ dia via oral \\
\hline Clopidogrel 75 mg & 75 mg/dia via oral \\
\hline Ácido Acetilsalicílico 100 mg & $100 \mathrm{mg} /$ dia via oral \\
\hline Anlodipino 5 mg & $10 \mathrm{mg} /$ dia via oral \\
\hline Levotiroxina 50 mcg & $50 \mathrm{mcg} /$ dia via oral \\
\hline Bromazepam 3 mg & $3 \mathrm{mg} /$ dia via oral \\
\hline
\end{tabular}


OLIVEIRA, R.E.M.; FILIPIN, M.D.V; GIARDIN, M.H.

REF-ISSN1808-0804 Vol.XII (2), 39-51, 2015.

Atorvastatina $\mathbf{2 0} \mathbf{~ m g}$

Omeprazol 20 mg

Losartana 25 mg

Insulina de Ação Intermediária

Insulina de Ação Rápida
$80 \mathrm{mg} /$ dia via oral

$20 \mathrm{mg} /$ dia via oral

$50 \mathrm{mg} /$ dia via oral

Uso subcutâneo:

40 UI - pela manhã

20 UI - antes de dormir

Uso subcutâneo:

4 UI - antes do café

2 UI - antes do jantar

\section{RESULTADOS E DISCUSSÃO}

A HAS é o principal fator de risco cardiovascular, especialmente para doenças coronarianas, acidente cerebrovascular e doença arterial periférica ${ }^{(8)}$. Apesar da existência de diversas classes de medicamentos disponíveis para a farmacoterapia da HAS, a falta de adesão à terapêutica ainda é uma preocupação, pois limita o êxito do tratamento ${ }^{(9)}$ e aumenta o risco de pressão arterial não controlada(10).

Neste relato, o resultado do teste BMQ demonstrou potencial baixa adesão, sendo constatadas barreiras relacionadas aos domínios regime, crenças e recordação. 0 paciente relatou dificuldades quanto ao acesso de medicamentos na rede pública de saúde, devido principalmente à indisponibilidade e consequentemente a necessidade de aquisição na rede privada. O mesmo referiu ser analfabeto e apresentar complicações na compreensão da prescrição médica e identificação de formas farmacêuticas, bem como horários de administração. Além disso, informou diminuição na acuidade visual e dificuldades no preparo e administração dos medicamentos nas doses corretas, dentre eles, a insulina. Portanto, aspectos intrínsecos deste paciente, como analfabetismo e idade, aliados à complexidade do regime terapêutico e à polifarmácia interferiram no processo de adesão do paciente ao tratamento. 
OLIVEIRA, R.E.M.; FILIPIN, M.D.V; GIARDIN, M.H.

REF-ISSN1808-0804 Vol.XII (2), 39-51, 2015.

Sabe-se que existem fatores de risco que podem influenciar negativamente a adesão e determinar diretamente o comportamento da pessoa frente ao tratamento medicamentoso da sua doença ${ }^{(11)}$. Dentre os principais fatores, estão os relacionados ao paciente e suas condições demográficas e sociais, à natureza da doença e características da terapêutica, ao sistema de saúde e o relacionamento do paciente com a equipe de profissionais e cuidadores $^{(9,10,12)}$.

A idade e o grau de escolaridade demonstraram ter participação fundamental na adesão ao esquema terapêutico. Um estudo realizado no sul do Brasil, que avaliou a adesão de indivíduos com 60 anos ou mais, revelou que cerca de $1 / 3$ dos idosos que utilizavam medicamentos eram não aderentes ao tratamento ${ }^{(13)}$. 0 diagnóstico de um maior número de morbidades relacionadas ao próprio processo de envelhecimento, associado à necessidade de tratamento simultâneo com vários fármacos podem contribuir consideravelmente para a baixa adesão do paciente idoso ${ }^{(14)}$.

Embora haja discussões na literatura quanto à influência do baixo grau de escolaridade na adesão, este deve ser classificado como um ponto relevante, pois está relacionado com as dificuldades na compreensão da prescrição médica e nos efeitos dos medicamentos, bem como na conscientização das doenças e da necessidade de tratamento ${ }^{(13,15)}$. Entretanto, para ser considerado um fator determinante, o grau de escolaridade deve ser avaliado de uma maneira ampla, também como um fator cultural, aliado a outras variáveis socioeconômicas, como renda familiar e ocupação(16). A análise destas variáveis no presente relato é uma limitação, pois não foi realizada.

A integralidade do cuidado durante a internação depende da atuação de uma equipe multidisciplinar e envolve a utilização de diversas tecnologias de saúde, bem como a execução de procedimentos técnicos e a criação de um ambiente confortável e seguro para o paciente hospitalizado. Neste contexto, está estruturada a função do farmacêutico, como uma referência na gestão da farmácia hospitalar e na atenção ao paciente ${ }^{(2)}$. 
OLIVEIRA, R.E.M.; FILIPIN, M.D.V; GIARDIN, M.H.

REF-ISSN1808-0804 Vol.XII (2), 39-51, 2015.

Neste estudo de caso, o farmacêutico analisou diversos aspectos da terapia farmacológica indicada ao paciente, como dosagem, posologia, interações e reações adversas. O seu atendimento junto ao paciente identificou as principais causas da baixa adesão ao tratamento e permitiu a proposta de estratégias para melhoria da mesma, como a disponibilização de ferramentas que auxiliam na organização e identificação dos medicamentos utilizados em domicílio e corresponsabilização de familiares nestes processos e também no acesso aos medicamentos.

Figura 2 - Principais etapas operacionais abordadas e desenvolvidas durante 0 acompanhamento farmacoterapêutico do paciente.

\section{Seguimento Farmacoterapêutico durante internação}

\section{Prescrição Médica}

- Conciliação Medicamentosa

- Avaliação das indicações dos medicamentos prescritos

- Avaliar necessidade de ajustes de doses e posologias

- Acompanhamento de exames laboratoriais

- Avaliação das vias de administrações e compatibilidades

- Interações Medicamentosas
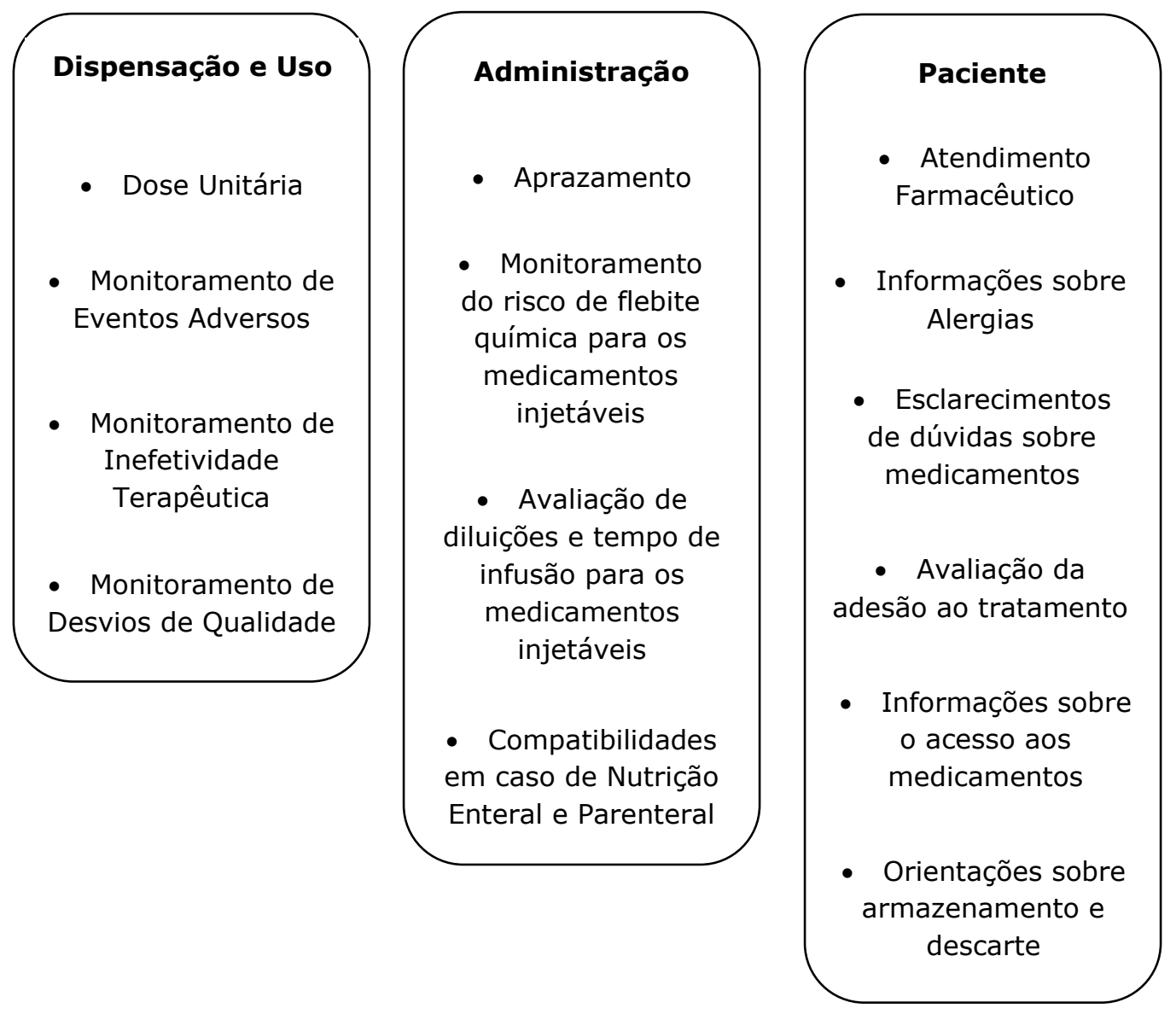
OLIVEIRA, R.E.M.; FILIPIN, M.D.V; GIARDIN, M.H.

REF-ISSN1808-0804 Vol.XII (2), 39-51, 2015.

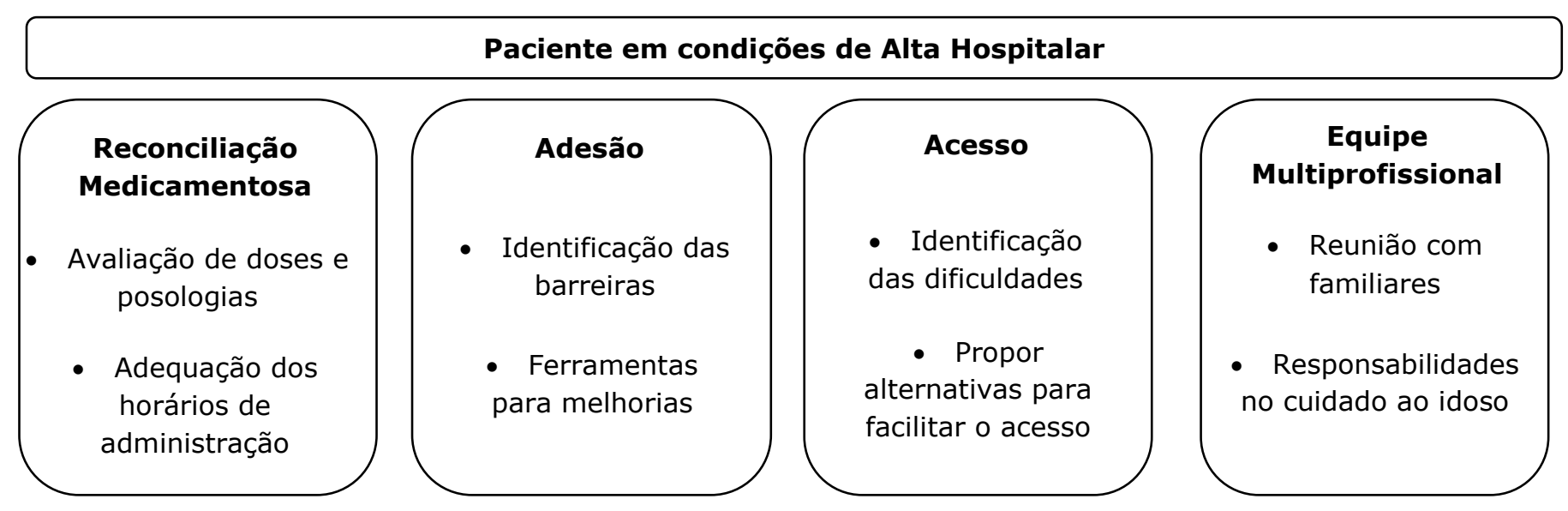

Figura 3 - Fluxograma operacional dos atendimentos do paciente às unidades hospitalares de atenção secundária e terciária e Estratégia Saúde da Família.

\section{Consulta no Ambulatório do Hospital de Atenção Terciária}

Encaminhamento médico com documentos de referência preenchidos.

2. Internação no Hospital de Atenção Secundária

Paciente recebe atendimento e realiza tratamento medicamentoso necessário até ter condições de receber alta hospitalar.

\section{Alta Hospitalar}

Agendamento da data para retorno no Ambulatório de Isquemia com documentos de contrarreferência preenchido.

\section{Retorno no Ambulatório do Hospital de Atenção Terciária}

Paciente retorna ao município de origem com documentos de contrarreferência e segue acompanhamento pela Atenção Primária à Saúde 
OLIVEIRA, R.E.M.; FILIPIN, M.D.V; GIARDIN, M.H.

REF-ISSN1808-0804 Vol.XII (2), 39-51, 2015.

\section{CONCLUSÃO}

A transição demográfica e epidemiológica brasileira tem atribuído importantes proporções ao número de comorbidades associadas, uso de múltiplos medicamentos e adesão ao tratamento enquanto desafio enfrentado por equipes de saúde cotidianamente.
Logo, fazem-se necessárias intervenções farmacêuticas em conjunto com a equipe multiprofissional no âmbito hospitalar com o objetivo de otimizar a adesão ao tratamento medicamento pósinternação. O contexto hospitalar não deve ser um ambiente destinado apenas à doença, mas um importante espaço de promoção e educação em saúde.

\section{REFERÊNCIAS}

1. CORRER, CJ et al. Riscos de problemas relacionados com medicamentos em pacientes de uma instituição geriátrica. Revista Brasileira de Ciências Farmacêuticas, 2007, v. 43, n. 1, p. 55-62.

2. ACURCIO, FA et al. Complexidade do regime terapêutico prescrito para idosos. Revista da Associação Médica Brasileira, 2009, v. 55, n. 4, p. 468-74.

3. LYRA-JÚNIOR, DP et al. A farmacoterapia no idoso: revisão sobre a abordagem multiprofissional no controle da hipertensão arterial sistêmica. Revista Latino-americana de Enfermagem. 2006, v. 14, n. 3, p. 428-34.

4. DOSSE, $C$ et al. Fatores associados à não adesão dos pacientes ao tratamento de hipertensão arterial. Rev. Latino-Am. Enfermagem [online]. 2009, vol.17, n.2 p. 201-06.

5. CASTRO, RA et al. Adesão ao tratamento de pacientes com insuficiência cardíaca em um hospital universitário. Rev. Gaúcha Enfermagem [online]. 2010, vol.31, n.2, p. 225-31.

6. BEN, AG et al. Teste de Morisky-Green e Brief Medication Questionnaire para avaliar adesão a medicamentos. Rev. Saúde Pública [online]. 2012, vol.46, n.2 p. 279-89.

7. Micromedex ${ }^{\circledR}$ Healthcare Series. Drug-Reax ${ }^{\circledR}$ System. Disponível no Portal CAPES em www.periodicos.capes.gov.br. Acessado em fevereiro de 2014.

8. A global brief on hypertension: silent killer, global public health crisis. World Health Day 2013. World Health Organization (WHO), Geneva 2013.

9. HAN E, et al. The impact of medication adherence on health outcomes for chronic metabolic diseases: A retrospective cohort study. Res Social Adm Pharm. 2014; doi: 10.1016/j.sapharm.2014.02.001. (Epub ahead of print).

10. GUSMÃo JL, et al. Adesão ao tratamento em hipertensão arterial sistólica isolada. Rev Bras Hipertens. 2009; 16(1): 38-43.

11. MARINKER M, SHAW J. Not to be taken as directed: putting concordance for taking medicines into practice. BMJ. 2003; 326: 348-349.

12. Adherence to long-term therapies - evidence for action. Noncommunicable diseases and mental health adherence to long term therapies project. World Health Organization (WHO), Geneva, 2003. 
OLIVEIRA, R.E.M.; FILIPIN, M.D.V; GIARDIN, M.H.

REF-ISSN1808-0804 Vol.XII (2), 39-51, 2015.

13. TAVARES NUL et al. Fatores associados à baixa adesão ao tratamento medicamentoso em idosos. Rev. Saúde Pública. 2013; 47(6): 1092-1101.

14. PASINA $L$ et al. Medication non-adherence among elderly patients newly discharged and receiving polypharmacy. Drugs Aging. 2014; 31 (4): 283-289.

15. GIROTTO E, et al. Adesão ao tratamento farmacológico e não farmacológico e fatores associados na atenção primária da hipertensão arterial. Ciênc. saúde coletiva. 2013; 18 (6): 1763-1772.

16. KIDD KE, ALTMAN DG. Adherence in social context. Control Clin Trials. 2000; 21 (5): 184S$187 \mathrm{~S}$. 